\title{
Immunohistochemical Study of Mitosis-regulatory Proteins in Gastroenteropancreatic Neuroendocrine Neoplasms
}

\author{
FRANZISKA BRIEST ${ }^{1,2}$, YAWEN WANG $^{1,3}$, RUZA ARSENIC $^{4}$, SEFER ELEZKURTAJ $^{4}$, \\ ERIKA BERG ${ }^{4}$, SONJA GRESHAKE $^{1}$, ADRIAN C. LOCK ${ }^{1}$, DIETER HÖRSCH ${ }^{2}$, CHRISTIAN N. ARNOLD $^{5}$, \\ MICHAEL HUMMEL ${ }^{4}$, BRITTA SIEGMUND ${ }^{1}$ and PATRICIA GRABOWSKI ${ }^{1,2,6}$ \\ Departments of ${ }^{1}$ Gastroenterology, Infectious Diseases and Rheumatology, ${ }^{4}$ Institute of Pathology, and \\ ${ }^{6}$ Medical Immunology, Charité - Universitätsmedizin Berlin, Corporate member of Freie Universität Berlin, \\ Humboldt-Universität zu Berlin and Berlin Institute of Health, Berlin, Germany; \\ ${ }^{2}$ Department of Gastroenterology and Endocrinology, Zentralklinik Bad Berka GmbH, Bad Berka, Germany; \\ ${ }^{3}$ Department of Neurosurgery, Vivantes Klinikum im Friedrichshain, Berlin, Germany; \\ ${ }^{5}$ Klinikum Friedrichshafen, Department of Gastroenterology, Friedrichshafen, Germany
}

\begin{abstract}
Background/Aim: Gastroenteropancreatic neuroendocrine neoplasms (GEP-NENs) are rare and heterogeneous tumors. Therapeutic targets remain to be identified and apart from the proliferation marker Ki-67, useful prognostic markers are rare. Mitotic proteins, such as forkheadbox protein M1 (FOXM1), survivin and aurora kinases, play a role in GEP-NEN progression. In this study, immunohistochemistry was used to analyze how this protein network is expressed in different subgroups of GEP-NENs and determine potential expression patterns that could be useful as tumor markers. Materials and Methods: Tumor tissues from 75 patients were studied immunohistochemically with antibodies against aurora B, survivin and FOXM1. The expression pattern was correlated with clinicopathological data such as tumor grading, metastatic state and prognosis. Results: The immunohistochemical analysis of nuclear aurora kinase $B$ revealed a positive correlation with nuclear survivin and FOXM1 staining patterns. Furthermore, aurora $B$ was positively related to grading and tumor size and negatively to differentiation and functionality. Conclusion: The expression of aurora kinase $B$ is associated with differentiation, progression and the aggressiveness of GEP-NENs. In the context of tumor
\end{abstract}

This article is freely accessible online.

Correspondence to: Franziska Briest, Charité-Universitätsmedizin Berlin, Department of Gastroenterology/Infectious Diseases/ Rheumatology, Hindenburgdamm 30, 12200 Berlin, Germany. Tel: +49 30450513824, e-mail: franziska.briest@ charite.de, ORCID ID: 0000-0001-5969-9467

Key Words: Aurora B, FOXM1, mitosis, chromosomal passenger complex, neuroendocrine tumors. progression, aurora $B$ is strongly associated with markers of the mitosis regulatory network, survivin, FOXM1 and Ki-67. A shift of the intracellular localization of aurora $B$ might be useful for the subclassification of intermediate-grade intestinal NET and NEC $(20 \%<K i-67<55 \%)$, since detailed Ki-67-based guidelines only exist for the pancreatic tumors. Most importantly, nuclear abundance of aurora $B$ was found to be strictly limited to high-grade tumors, which is important for the consideration of aurora inhibitors for therapy of NENs.

The heterogeneous group of neuroendocrine neoplasms (NENs) of the pancreas and the gastrointestinal tract (GEP) comprise of slowly growing well-differentiated tumors, as well as highly proliferative poorly differentiated carcinomas. Recent classification systems distinguish well-differentiated grade $(\mathrm{G}) 1$ and $\mathrm{G} 2$ tumors by use of the Ki-67 proliferation marker indices of $\leq 2 \%$ and $\leq 20 \%$, respectively (1). G3 tumors, formerly characterized by Ki-67>20\% are increasingly subdivided by morphological and prognostic criteria, resulting in two groups: G3 well-differentiated neuroendocrine tumors (NET) and G3 poorly differentiated neuroendocrine carcinomas (NEC) (2). According to the WHO 2017 guidelines for pancreatic NET classification, G3 NEC are further subdivided into small and large cell subtypes (3). A Ki-67 cut-off of $\geq 55 \%$ has been proposed to distinguish G3 NET and NEC based on the Nordic NEC study $(3,4)$. The present study analyzed the expression of potential proliferation markers associated with mitotic cell activity in well-differentiated NEN and in comparison with G3 NEC.

The transcription factor forkheadbox protein M1 (FOXM1) controls crucial mechanisms in cancer cells. By determining $\mathrm{G}_{1} / \mathrm{S}$ and $\mathrm{G}_{2} / \mathrm{M}$ progression of the cell cycle, it is involved in tumor growth (5). It contributes to the regulation of vascular endothelial growth factor (VEGF) and thereby to the 
Table I. Histopathological data of the analyzed patients. All G3 tumors were morphologically evaluated. All G3 tumors with Ki-67 $\geq 55 \%$ were verified as poorly differentiated. One G3 tumor with Ki-67<55\% had mixed differentiation.

\begin{tabular}{|c|c|c|c|c|c|c|}
\hline & & \multicolumn{5}{|c|}{ Grading WHO 2017, n } \\
\hline & & G1 NET & G2 NET & $\begin{array}{c}\text { G3 NET/NEC } \\
\text { Ki67<55\% }\end{array}$ & $\begin{array}{c}\text { G3 NEC } \\
\text { Ki67 } \geq 55 \%\end{array}$ & Total \\
\hline \multirow[t]{3}{*}{ Tisssue type } & Primary & 38 & 18 & 2 & 7 & 65 \\
\hline & MTS & 5 & 5 & 0 & 0 & 10 \\
\hline & Total & 43 & 23 & 2 & 7 & 75 \\
\hline \multirow[t]{6}{*}{ Localization of the primary } & Gastric & 2 & 2 & 0 & 1 & 5 \\
\hline & Pancreatic & 7 & 7 & 0 & 0 & 14 \\
\hline & Small intestinal & 25 & 12 & 1 & 0 & 38 \\
\hline & Appendix & 6 & 0 & 0 & 0 & 6 \\
\hline & Large intestinal & 3 & 2 & 1 & 6 & 12 \\
\hline & Total & 43 & 23 & 2 & 7 & 75 \\
\hline \multicolumn{7}{|l|}{ Metastasis status } \\
\hline & M0 & 23 & 7 & 1 & 0 & 31 \\
\hline & M1 & 16 & 14 & 1 & 7 & 38 \\
\hline & $\mathrm{Mx}$ & 4 & 2 & 0 & 0 & 6 \\
\hline & Total & 43 & 23 & 2 & 7 & 75 \\
\hline Average age, years & & 54.57 & 56.87 & 50.50 & 53.86 & 55.11 \\
\hline \multirow[t]{3}{*}{ Gender } & Male & 20 & 8 & 2 & 6 & 36 \\
\hline & Female & 23 & 15 & 0 & 1 & 39 \\
\hline & Total & 43 & 23 & 2 & 7 & 75 \\
\hline
\end{tabular}

MTS: Metastases.

vascularization of malignant tissue (6). It supports metastasis by up-regulation of extracellular matrix remodeling enzymes, destabilization of cytoskeleton structures, stimulation of the generation of pre-metastatic niche and induction of epithelialmesenchymal transformation $(7,8)$. Furthermore, FOXM1 has been demonstrated to regulate DNA repair and thereby confers resistance to chemotherapy (9). Finally, we previously demonstrated that FOXM1 is involved in progression of NETs of the intestine and the lung $(10,11)$.

FOXM1 regulates genes that contribute to control of mitosis $(5,12)$. These include proteins of the chromosomal passenger complex, especially aurora kinase B and survivin (13). The chromosomal passenger complex orchestrates the proper segregation of chromosomes and cytokinesis. Furthermore, recent studies suggest that survivin is not only involved in cellular proliferation, but also contributes to DNA-damage response, apoptosis inhibition and induction of cellular senescence (14-16). Although previous studies have demonstrated a positive association of high FOXM1 and survivin expression with grading in GEP-NEN $(10,17)$, to our knowledge, the role of aurora kinase B has not been elucidated.

\section{Materials and Methods}

Patients and samples. Seventy-five formalin-fixed and paraffinembedded tissue specimens of NETs collected between 1982 and 2006 at the Charité Universitätsmedizin Berlin, the University
Hospital Marburg and the University Hospital Freiburg, Germany, were enrolled in this study after approval of the local Ethical Committee (EA1/321/16). Clinicopathological data including Ki-67 and follow-up data (survival) for all patients were available. Inclusion criteria were positive staining for neuroendocrine markers (chromogranin A, synaptophysin A) and availability of clinicopathological information. Tumors were re-classified according to the WHO 2010 (non-pancreatic) and WHO 2017 (pancreatic) classification $(18,19)$. G3 Gastrointestinal neoplasms were further separated by morphological criteria and with a Ki-67 cut-off at $\geq 55 \%$ (4). Two G3 tumors were diagnosed with Ki-67 of between $20 \%$ and $55 \%$; one tumor showed a mixed morphology with highly and poorly differentiated clusters, the second tumor showed an intermediate to poor differentiation. The neoplasms with Ki-67 $\geq 55 \%$ were undifferentiated carcinomas, including two smallcell NECs. Clinicopathological data can be found in Table I.

Immunohistochemistry. Paraffin sections were stained with the following antibodies using the standard avidin-biotin-peroxidase kit (DAKO Hamburg, Germany): Aurora B/AIM-1: N=75 (611082; BD Transduction Laboratories, San Jose, CA, USA), survivin: N=75 (ab469; Abcam, Cambridge, UK). Avidin-Biotin method (20) was used for staining with anti-FOXM1: N=30 (sc-32855; Santa Cruz Inc., Dallas, TX, USA). Antibodies were diluted to a working concentration (aurora B: 1:50; FOXM1: 1:100; survivin: 1:4000).

Staining was evaluated independently by two pathological experts (R.A. and S.E.) as follows: Nuclear aurora B staining: Low: $<5 \%$ of cells, high: $>5 \%$ of cells. Cytosolic aurora B was determined as negative and positive without further specification. Survivin and FOXM1 were quantitated as previously published (10, 
Table II. Tabulation of immunohistochemical staining results for aurora B, survivin and forkheadbox protein M1 (FOXM1) in relation to tumor grading. All scores were significantly related to grading in univariate analyses. FOXM1 and nuclear survivin were further significantly associated with grading in a multivariate analysis.

\begin{tabular}{|c|c|c|c|c|c|c|c|}
\hline \multirow[b]{2}{*}{ Grading } & \multicolumn{4}{|c|}{$\mathrm{G} 3, \mathrm{n}(\%)$} & \multirow[b]{2}{*}{ Total, $\mathrm{n}$} & \multicolumn{2}{|c|}{$p$-Value } \\
\hline & G1 NET, n (\%) & G2 NET & Ki-67<55\% & $\mathrm{Ki}-67 \geq 55 \%$ & & Univariate & Multivariate \\
\hline Cytosolic aurora B & $33 / 43(76.7 \%)$ & $14 / 23(60.9 \%)$ & $0 / 2(0.0 \%)$ & $1 / 7(14.3 \%)$ & 75 & 0.003 & 0.169 \\
\hline Nuclear aurora B & $2 / 43(4.7 \%)$ & $0 / 23(0.0 \%)$ & $1 / 2(50.0 \%)$ & $6 / 7(85.7 \%)$ & 75 & $<0.001$ & 0.554 \\
\hline Nuclear survivin & $0 / 43(0.0 \%)$ & $4 / 23(17.4 \%)$ & $2 / 2(100.0 \%)$ & $7 / 7(100.0 \%)$ & 75 & $<0.001$ & $<0.001$ \\
\hline FOXM1 & $0 / 15(0.0 \%)$ & $2 / 8(25.0 \%)$ & $0 / 1(0.0 \%)$ & $3 / 6(50.0 \%)$ & 30 & 0.032 & 0.034 \\
\hline
\end{tabular}

NET: Neuroendocrine tumor.

$17,21)$. Briefly, Survivin was considered positive if $>5 \%$ of the nuclei stained positive; FOXM1 was determined by including cytoplasmic (intensity: 0-1 low, 2-3 high) and nuclear staining (percentage of positive cells $0 \%=0 ; 1-10 \%=1,11-50 \%=2,>50 \%=3$ was multiplied by intensity: negative $=0 ;$ light $=1$; strong $=2$. Nuclear FOXM1 was considered high with a resulting score $\geq 4$ ). Overall FOXM1 staining was defined high when both scores were high.

Statistical evaluation. For univariate analyses, the chi-square test was applied. For results with low expected counts due to low sample numbers, Fisher's exact test was applied. Multivariate analysis was conducted by forward and backward linear regression. Co-variates included were metastatic status and the results of all immunohistochemical stainings. Survival was evaluated by the MantelCox log-rank test and in a multivariate analysis by Cox regression (enter and backward stepwise regression method) according to grading and metastatic status. Differences were considered significant for $p<0.05$. All statistical analyzes were performed using IBM SPSS Statistics v22 software (IBM, Armonk, NY, USA) and further visualized by Prism v7 (Graphpad Software, La Jolla, CA, USA).

\section{Results}

Nuclear immunoreactivity of aurora B, survivin and FOXMI are associated with grading and differentiation of GEP-NEN. Immunohistochemical analysis of 75 GEP-NEN samples demonstrated a high percentage of aurora B-positive nuclei in high-grade NECs $\geq 55 \% \mathrm{Ki}-67$, whereas well-differentiated G1/2 and G3 tumors with Ki- $67<55 \%$ showed virtually no nuclear aurora B positivity (Figure 1). In contrast, the cytosolic aurora B score was high in well-differentiated G1G2 tumors and low in G3 NECs with $\mathrm{Ki}-67 \geq 55 \%$.

High positivity of FOXM1 (including high nuclear and high cytosolic staining) (10)) and high nuclear survivin were positively related to high grading and low differentiation, as both were low in G1 and G2, and high in G3 NECs (Figure $2)$. These results are in accordance with already published data of our group $(10,17)$. Concluding, all studied mitotic proteins showed nuclear immunoreactivity to increase with increasing Ki-67 index of the tumors (Table II).
Nuclear immunoreactivity of aurora $B$ is associated with expression of other mitotic proteins, inversely related to cytosolic aurora $B$ and correlated with tumor size and functionality. All nuclear scores were strongly correlated with each other; nuclear scores for aurora B and survivin were inversely associated with the cytosolic aurora B immunoreactivity (Table III). Finally, nuclear aurora B, survivin and high total FOXM1 immunoreactivity were related to a high tumor volume (Table IV) and positive nuclear aurora B and survivin were significantly increased in non-functional tumors (Table V).

Aurora B expression is not suitable as independent prognostic marker. The analysis of the patient survival showed a univariate significant association of aurora B nuclear staining with survival after the log-rank (Mantel-Cox) test (Figure 3). Nevertheless, as nuclear aurora B is highly associated with tumor grading, these results were expected not to be independent from tumor grading as covariate. The Cox regression statistics including metastasis status and tumor grading confirmed this assumption, aurora B was not validated as an independent prognostic factor. Therefore, aurora B does not have independent prognostic significance.

\section{Discussion}

Mitotic proteins not only control cell-cycle progression and proper chromosomal and cellular separation, but are considered important signaling mediators and potential targets of anticancer therapeutics. Whereas cell signalregulatory functions of $\mathrm{Ki}-67$ are almost unexplored, the mitotic orchestrator FOXM1, which controls the transcription of the genes of aurora kinases A and B (AURKA and $A U R K B)$, survivin (BIRC5) and Ki-67 (MKI-67) among many others, has been described as a crucial determiner of many cancer-supporting mechanisms. These include angiogenesis, metastasis, DNA-damage repair, therapy resistance and cell-cycle progression $(5,6)$. Furthermore, 

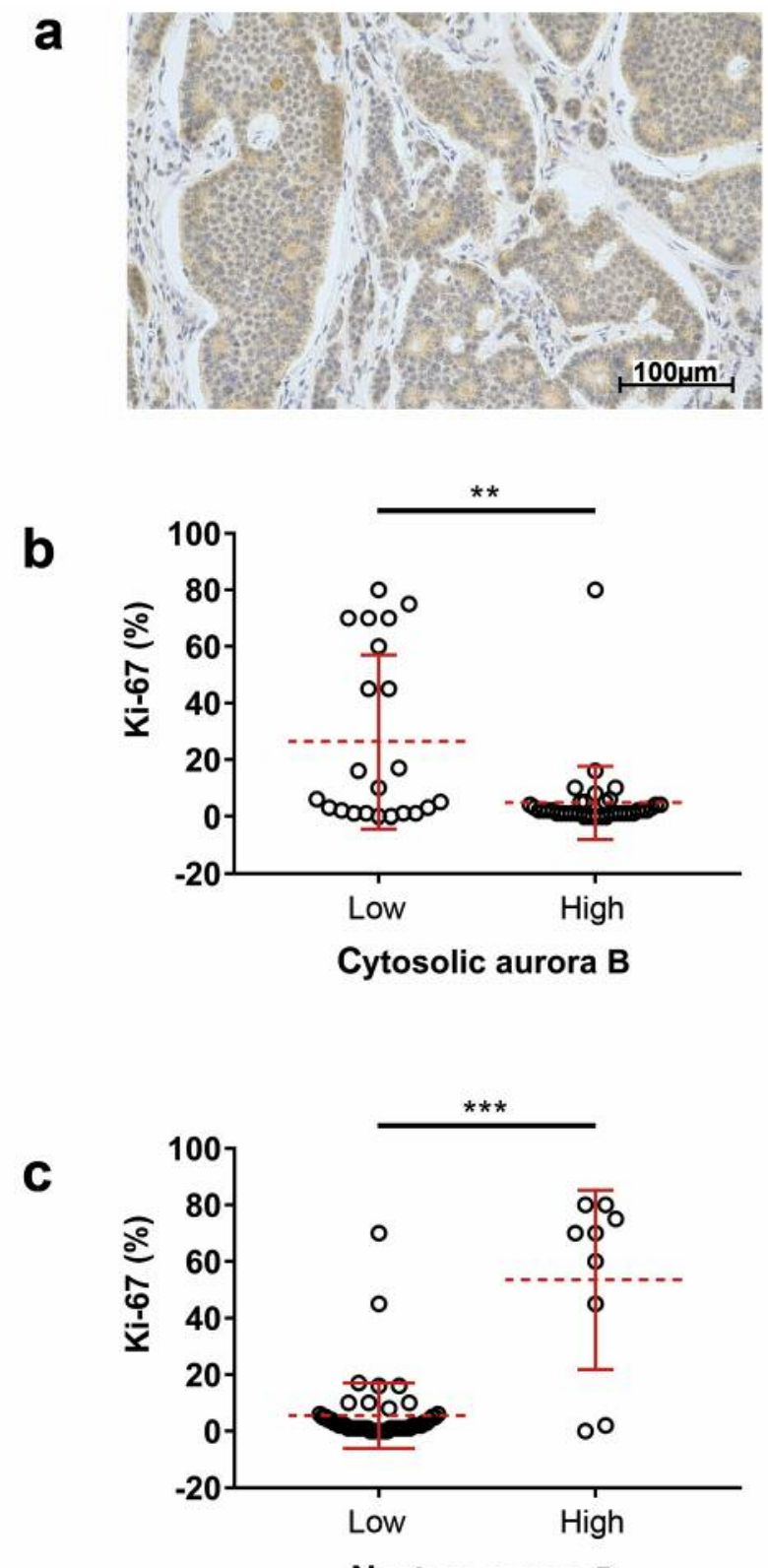

Nuclear aurora B
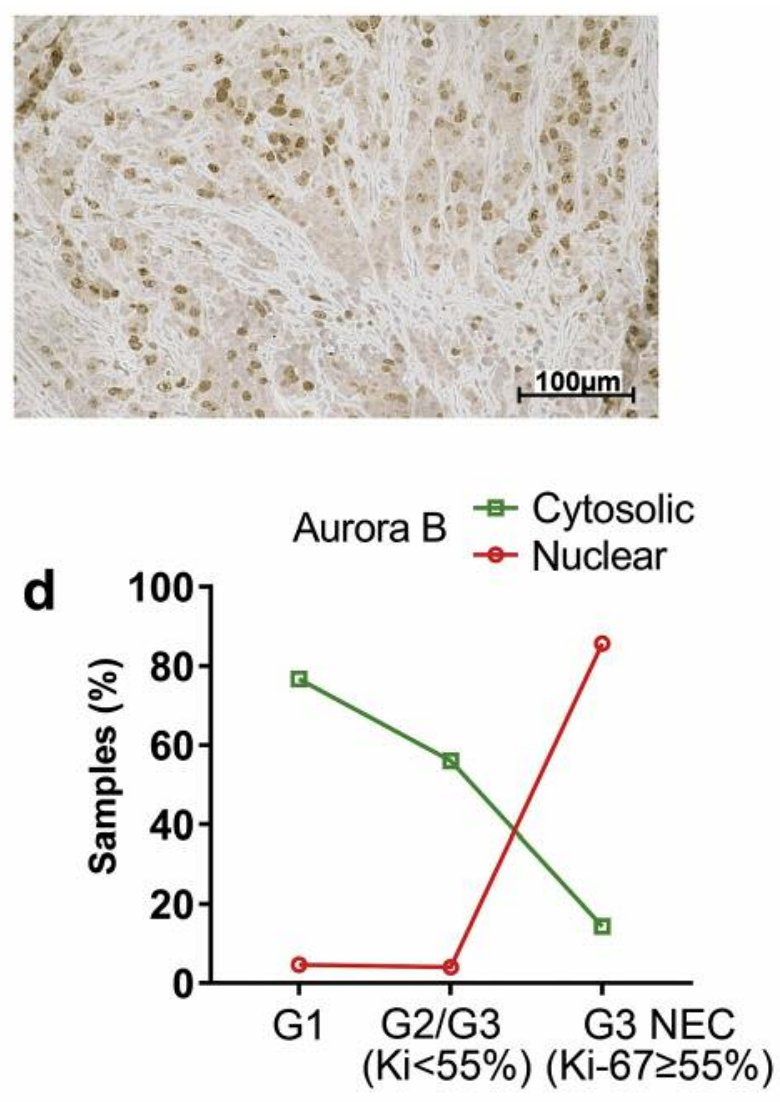

NET

Figure 1. Results of immunohistochemical staining of aurora $B$ in gastroenteropancreatic neuroendocrine neoplasms (GEP-NEN). High cytosolic immunoreactivity [a: left panel $(\times 100)$ and b] was associated with a low Ki-67 index $(p=0.0058)$, whereas a high nuclear score [a: right panel $(\times 400)$ and $c]$ was correlated with a high Ki-67 index $(p=0.0006)$. Both scores were therefore inversely related to each other ( $d ; p=0.001)$. Significantly different at $* p<0.05, * * p<0.01, * * * p<0.001$, and $* * * *<<0.0001$. NEN: Neuroendocrine neoplasms; NEC: neuroendocrine carcinoma.

high expression of FOXM1 was recently associated with aggressiveness in several neuroendocrine cancer entities (10, 11). Consistently, the expression of FOXM1 targets survivin and aurora A have been related to GEP NEN grading (17, 22). Previous work also analyzed the expression of aurora $B$ in pancreatic and small intestinal NENs and concluded that positive immunoreactivity was infrequent (22). In this study, however, only one high-grade tumor was included, and this G3 pancreatic tumor was aurora B-positive. As only two out of $66 \mathrm{G} 1$ and G2 NENs in our study exhibited positive staining, our data are consistent with this previous work, but add further information about the role of aurora kinase B in high-grade tumors. According to our results, high nuclear aurora B abundance was related to a high tumor grading, which was characterized not only by a high Ki-67 value, but also by a large tumor size and a loss of tumor functionality, which are typical characteristics of high-grade NETs.

The aurora kinase inhibitor danusertib has been shown to be effective in BON and QGP-1 cells in vitro and in an orthotopic xenograft pancreatic NET model (22). Our results 
a
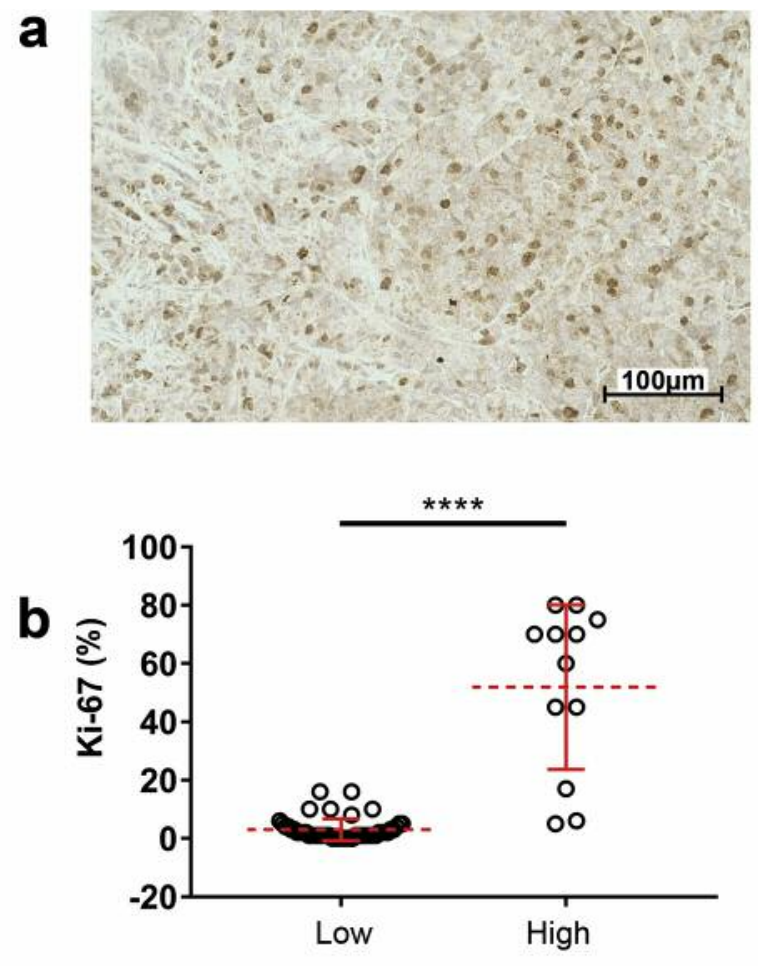

Nuclear survivin

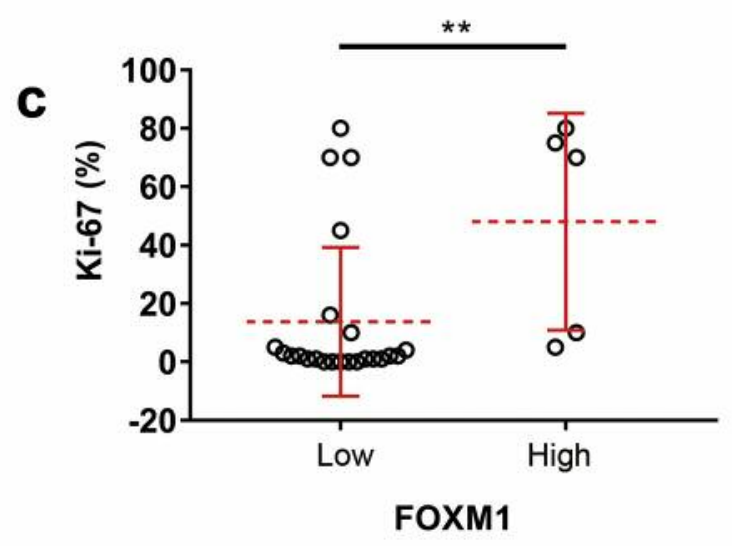

indicate that aurora $\mathrm{B}$ inhibition might be even more beneficial in high-grade tumors and carcinomas than in the analyzed cell lines, which were generated from welldifferentiated primary material. Nevertheless, the untypical mutation patterns of both cell lines $(23,24)$ brings into question whether these cell lines represent well-differentiated tumors. Furthermore, a variety of aurora inhibitors have already been made available (25).

An interesting finding was the inversely associated immunoreactivity of cytosolic and nuclear aurora B. A transcriptional dependency of aurora B on FOXM1 as
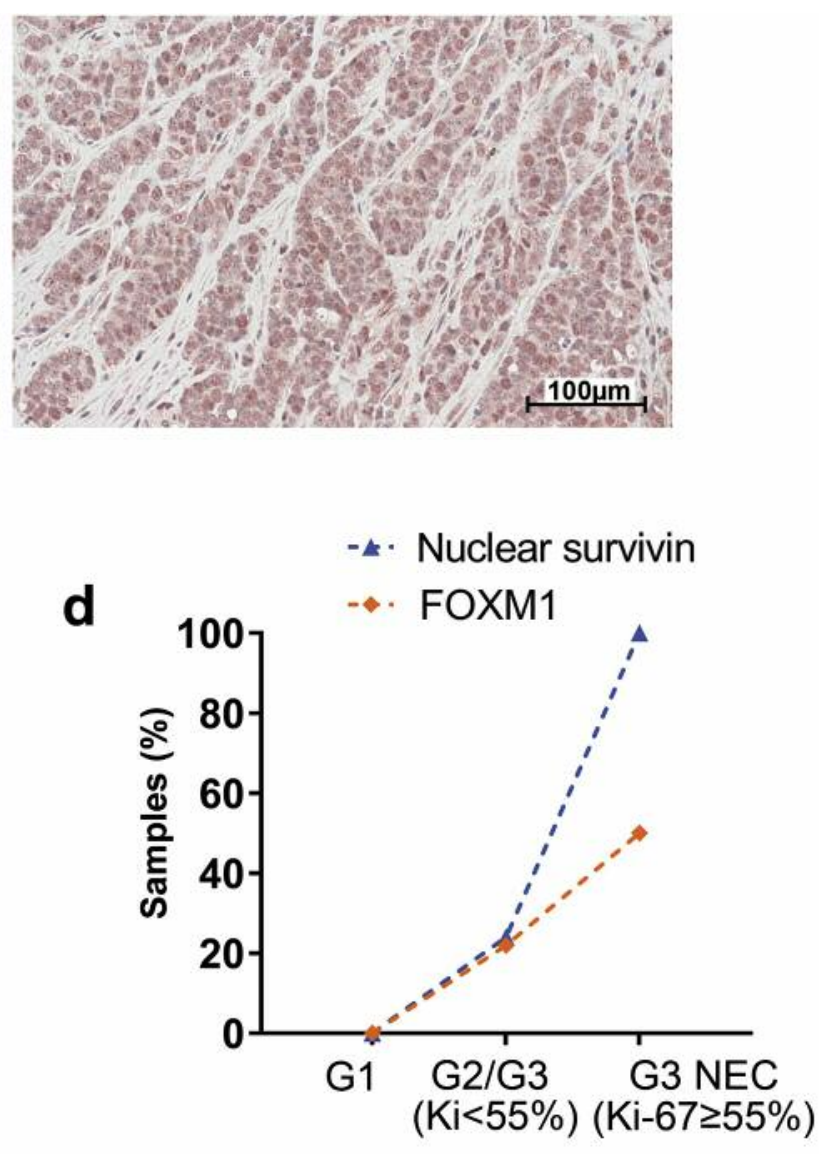

NET

Figure 2. Results of immunohistochemical staining of nuclear survivin and forkheadbox protein M1 (FOXM1) in gastroenteropancreatic neuroendocrine neoplasms (GEP-NEN). High nuclear score for survivin [a: left panel $(\times 100)$ and $b p<0.0001]$ and a high general FOXM1 expression [a: right panel $(\times 100)$ and $c ; p=0.009]$ were correlated with a high Ki 67 staining of the tumor samples. Both FOXM1 and nuclear survivin reactivity (d) were very high in the G3 neuroendocrine carcinoma (NEC) subgroup. Significantly different at $* p<0.05$, $* * p<0.01, * * * p<0.001$, and $* * * * p<0.0001$. Light microscopy, corresponding specimens of one G3 NEC.

described in the literature (13) would suggest de novo protein expression following FOXM1 transcription factor activity. Nevertheless, we showed here that low-grade tumors (with low FOXM1 expression) have high amounts of aurora B, but the protein was localized in the cytoplasm. There are two possible explanations for this: Aurora B was either exported from the nucleus or it was not translocated into the nucleus after its synthesis. Consequently, there is a post-translational aurora Bregulatory mechanism similar to the nucleocytoplasmic shuttling of survivin, which regulates the latter's antiapoptotic regulatory activity $(16,26)$. 


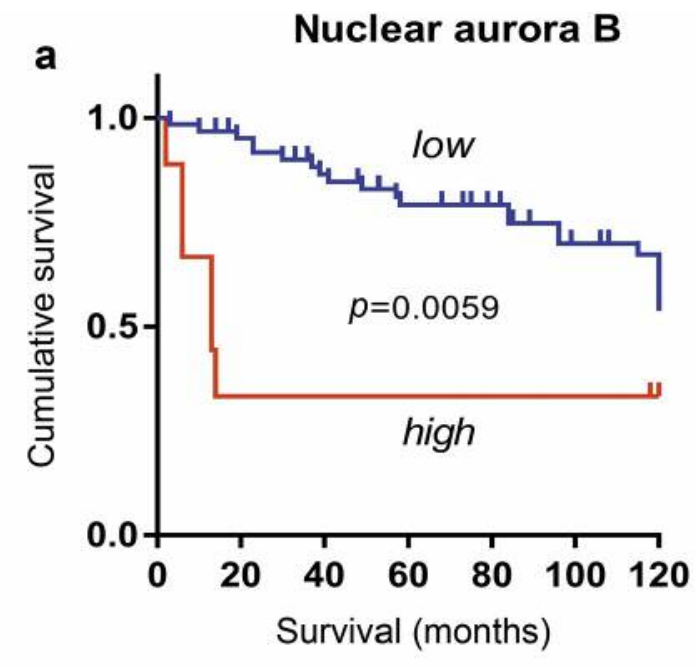

b

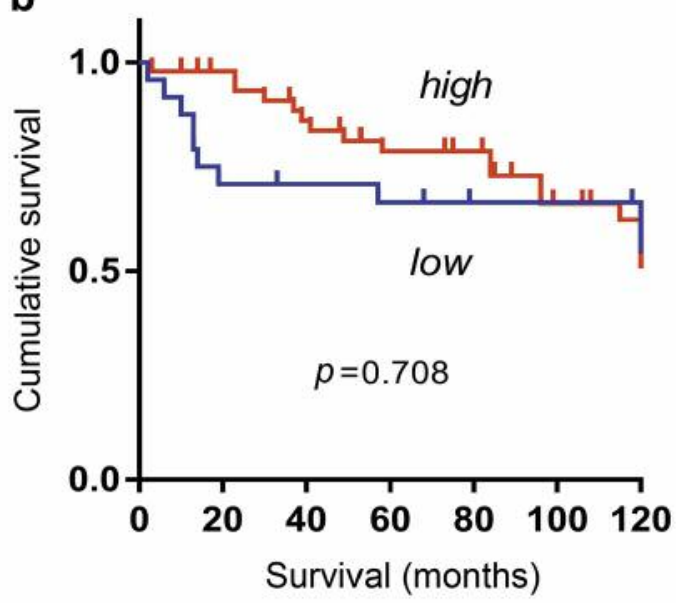

C
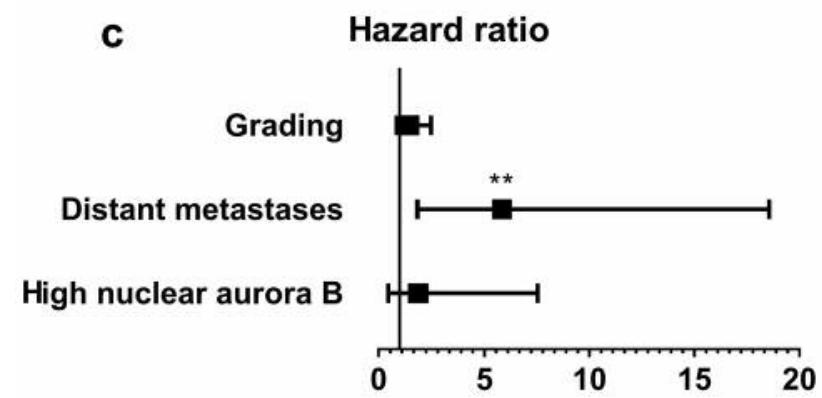

Figure 3. Kaplan-Meier survival curves for patients with high nuclear or cytosolic aurora B expression. Univariate results of cumulative survival analysis with nuclear (a) and cytosolic (b) aurora B localization as an independent variate. -Values were calculated by the log-rank test. Hazard ratios with 95\% confidence intervals (c) of the analyzed co-variates in the multivariate Cox-regression analysis using Enter method are depicted. The hazard ratio estimates an average $>5$-fold risk for tumor-related death within the observed period if patients had distant metastases (5-and 10year overall survival (OS) for $M=0$ was $82 \%$ and $64 \%$, respectively; 5 and 10-year OS for $M=1$ was $47 \%$ and $16 \%$, respectively). The covariates of grading and high nuclear aurora B showed a risk between 1-fold and 2 -fold and are therefore not predictive. Significantly different at ${ }^{* *} p<0.01$.
Table III. Tabulation of the correlation of immunohistochemical staining results for aurora B, survivin and forkheadbox protein M1 (FOXM1). Cytosolic aurora B staining was inversely associated with nuclear aurora B and survivin immunoreactivity. High FOXM1 and nuclear survivin were positively correlated with nuclear aurora B staining (univariate analyses).

\begin{tabular}{lccc}
\hline & Nuclear aurora B & Nuclear survivin & FOXM1 \\
\hline Cytosolic aurora B & $\mathrm{n}=75, p=0.001$ & $\mathrm{n}=75, p=0.000$ & $\mathrm{n}=30$, \\
& (inverse) & (inverse) & $p=0.143$ \\
Nuclear aurora B & & $\mathrm{n}=75$, & $\mathrm{n}=30$, \\
& & $p<0.001$ & $p=0.041$ \\
\hline
\end{tabular}

Table IV. Tabulation of the immunohistochemical staining results for aurora B, survivin and forkheadbox protein M1 (FOXM1) in relation to tumor size. High expression of FOXM1, nuclear aurora B and nuclear survivin was significantly more frequent in large tumors.

\begin{tabular}{lllll}
\hline & \multicolumn{3}{c}{ Tumor size, $\mathrm{n}(\%)$} & \\
\cline { 2 - 3 } Marker & Small $(<2 \mathrm{~cm})$ & Large $(\geq 2 \mathrm{~cm})$ & Total, $\mathrm{n}$ & $p$-Value* \\
\hline Nuclear aurora B & $0 / 30(0.0 \%)$ & $8 / 24(33.3 \%)$ & 54 & 0.001 \\
Nuclear survivin & $3 / 30(10.0 \%)$ & $8 / 24(33.3 \%)$ & 54 & 0.034 \\
FOXM1 & $0 / 11(0.0 \%)$ & $5 / 15(33.3 \%)$ & 26 & 0.033 \\
\hline
\end{tabular}

*Univariate.

Table V. Tabulation of the immunohistochemical staining results for nuclear aurora $B$, and survivin in relation to functionality of the tumors. Non-functional tumor significantly more frequently expressed nuclear survivin and aurora $B$.

\begin{tabular}{|c|c|c|c|c|}
\hline \multirow[b]{2}{*}{ Marker } & \multicolumn{2}{|c|}{ Functionality, n (\%) } & \multirow[b]{2}{*}{ Total, n } & \multirow[b]{2}{*}{$p$-Value* } \\
\hline & Functional & Non-functional & & \\
\hline Nuclear aurora B & $0 / 23(0.0 \%)$ & $8 / 34(23.5 \%)$ & 57 & 0.016 \\
\hline Nuclear survivin & $1 / 23(4.3 \%)$ & $9 / 34(26.5 \%)$ & 57 & 0.031 \\
\hline
\end{tabular}

*Univariate.

According to recent literature there is evidence that a concentration gradient exists between the nuclear and cyctosolic pools of aurora B. This determines the activity of the mitotic regulator by autophosphorylation (27). Furthermore, a nuclear export factor chromosomal maintenance 1 (CRM1) has been described to direct the nuclear export of aurora kinases (28). Whether the cytoplasmic pool of aurora B has an inherent signaling function, or the protein is simply stored in the centrosomes remains to be further elucidated. Interestingly, a previous 
article reported that aurora B (in contrast to aurora A) was predominantly located in the nucleus during interphase (28). However, in our study we found the complete opposite, with significant results. Therefore, the nucleocytoplasmic localization of aurora B during the non-mitotic cell-cycle phases might be dependent on the cell type.

The expression of mitotic protein $\mathrm{Ki}-67$, but also of FOXM1, survivin and aurora kinases, increases with the aggressiveness of the tumors. Especially in G3 tumors, the wide range of $\mathrm{Ki}-67$ from $20 \%$ to $100 \%$ indicates the need for further subclassification. Our data reflect the need for inclusion of morphological as well as Ki-67-based criteria for G3 subclassification, as proposed by Fazio and colleagues in 2016, who proposed a general introduction of G4 NEC when Ki-67 exceeds 55\%, and a subclassification into G3 NET and G3 NEC for Ki-67 values of between $20 \%$ and $55 \%$ (29). In accordance with this, we found heterogeneous results for two tumors with $20 \%$ to $55 \%$ Ki67 , irrespective of differentiation. However, the number of G3 tumors with Ki-67 $<55 \%$ was much too small for a reliable conclusion to be drawn for this subgroup.

Summarizing, this study presents the association of high nuclear aurora B immunoreactivity with the nuclear presence of other mitotically-related proteins and a high tumor grade. A regulatory effect of nucleocytoplasmic localization remains to be further investigated. As aurora B is present in highly proliferating NEN cells, further preclinical testing of aurora kinase inhibitors should be considered in high-grade GEP-NET and-NEC rather than in G1 or G2 NETs.

\section{Funding}

F.B. and P.G. were supported by the Theranostic Research Network Germany. The other Authors state no relevant funding.

\section{Conflicts of Interest}

The group of Dr. Patricia Grabowski received financial support from Ipsen Pharma, Novartis and Pfizer. F.B. and P.G. received a travel grant and a lecture salary from Ipsen Pharma. Y.W. received a travel grant from Novartis.

\section{References}

1 Rindi G, Arnold R, Bosman F, Capella C, Klimstra D, Klöppel G, Komminoth P and Solcia E: 2010 Nomenclature and classification of neuroendocrine neoplasms of the digestive system. In: WHO Classification of Tumours of the Digestive System (Bosman F, Carneiro F, Hruban R and Theise N (eds.)). Lyon, IARC Press, pp. 13-14, 2010.

2 Schimmack S, Svejda B, Lawrence B, Kidd M and Modlin IM: The diversity and commonalities of gastroenteropancreatic neuroendocrine tumors. Langenbecks Arch Surg 396(3): 273298, 2011.

3 Klöppel G, Klimstra D, Hruban R, Adsay V, Capella C, Couvelard A, Komminoth P, La Rosa S, Ohike N, Osamura R, Perren A,
Scoazec J and Rindi G: Pancreatic neuroendocrine tumors: Update on the New World Health Organization Classification. AJSP: Reviews and Reports 22(5): 233-239, 2017.

4 Sorbye H, Welin S, Langer SW, Vestermark LW, Holt N, Osterlund P, Dueland S, Hofsli E, Guren MG, Ohrling K, Birkemeyer E, Thiis-Evensen E, Biagini M, Gronbaek H, Soveri LM, Olsen IH, Federspiel B, Assmus J, Janson ET and Knigge $\mathrm{U}$ : Predictive and prognostic factors for treatment and survival in 305 patients with advanced gastrointestinal neuroendocrine carcinoma (WHO G3): the NORDIC NEC study. Ann Oncol 24(1): 152-160, 2013.

5 Wierstra I: FOXM1 (Forkhead box M1) in tumorigenesis: overexpression in human cancer, implication in tumorigenesis, oncogenic functions, tumor-suppressive properties, and target of anticancer therapy. Adv Cancer Res 119: 191-419, 2013.

6 Zhang Y, Zhang N, Dai B, Liu M, Sawaya R, Xie K and Huang $\mathrm{S}$ : FoxM1B transcriptionally regulates vascular endothelial growth factor expression and promotes the angiogenesis and growth of glioma cells. Cancer Res 68(21): 8733-8742, 2008.

7 Halasi M and Gartel AL: FOX(M1) news - it is cancer. Mol Cancer Ther 12(3): 245-254, 2013.

8 Park HJ, Gusarova G, Wang Z, Carr JR, Li J, Kim KH, Qiu J, Park YD, Williamson PR, Hay N, Tyner AL, Lau LF, Costa RH and Raychaudhuri P: Deregulation of FoxM1b leads to tumour metastasis. EMBO Mol Med 3(1): 21-34, 2011.

9 Zona S, Bella L, Burton MJ, Nestal de Moraes G and Lam EW: FOXM1: an emerging master regulator of DNA damage response and genotoxic agent resistance. Biochim Biophys Acta 1839(11): 1316-1322, 2014.

10 Briest F, Berg E, Grass I, Freitag H, Kaemmerer D, Lewens F, Christen F, Arsenic R, Altendorf-Hofmann A, Kunze A, Sänger J, Knösel T, Siegmund B, Hummel $M$ and Grabowski P: FOXM1: A novel drug target in gastroenteropancreatic neuroendocrine tumors. Oncotarget 6(10): 8185-8199, 2015.

11 Taromi S, Lewens F, Arsenic R, Sedding D, Sänger J, Kunze A, Möbs M, Benecke J, Freitag H, Christen F, Kaemmerer D, Lupp A, Heilmann M, Lammert H, Schneider C, Richter K, Hummel M, Siegmund B, Burger M, Briest F and Grabowski P: Proteasome inhibitor bortezomib enhances the effect of standard chemotherapy in Small Cell Lung Cancer. Oncotarget 8: 97061-97078, 2017.

12 Wierstra I: The transcription factor FOXM1 (Forkhead box M1): proliferation-specific expression, transcription factor function, target genes, mouse models, and normal biological roles. Adv Cancer Res 118: 97-398, 2013.

13 Wang IC, Chen YJ, Hughes D, Petrovic V, Major ML, Park HJ, Tan Y, Ackerson T and Costa RH: Forkhead box M1 regulates the transcriptional network of genes essential for mitotic progression and genes encoding the SCF (Skp2-Cks1) ubiquitin ligase. Mol Cell Biol 25(24): 10875-10894, 2005.

14 Unruhe B, Schröder E, Wünsch D and Knauer SK: An Old Flame Never Dies: Survivin in Cancer and Cellular Senescence. Gerontology 62(2): 173-181, 2016.

15 Hoffman WH, Biade S, Zilfou JT, Chen J and Murphy M: Transcriptional repression of the anti-apoptotic survivin gene by wild type p53. J Biol Chem 277(5): 3247-3257, 2002.

16 Knauer SK, Kramer OH, Knösel T, Engels K, Rödel F, Kovács AF, Dietmaier W, Klein-Hitpass L, Habtemichael N, Schweitzer A, Brieger J, Rödel C, Mann W, Petersen I, Heinzel T and Stauber RH: Nuclear export is essential for the tumor-promoting activity of survivin. FASEB J 21(1): 207-216, 2007. 
17 Grabowski P, Griss S, Arnold CN, Hörsch D, Göke R, Arnold R, Heine B, Stein H, Zeitz M and Scherübl H: Nuclear survivin is a powerful novel prognostic marker in gastroenteropancreatic neuroendocrine tumor disease. Neuroendocrinology 81(1): 1-9, 2005.

18 Klöppel G: Classification and pathology of gastroenteropancreatic neuroendocrine neoplasms. Endocr Relat Cancer 18(Suppl 1): S1-16, 2011.

19 Klöppel G, Klimstra D, Hruban R, Adsay V, Capella C, Couvelard A, Komminoth P, La Rosa S, Ohike N, Osamura R, Perren A, Scoazec J and Rindi G: Pancreatic Neuroendocrine Tumors: Update on the New World Health Organization Classification. AJSP: Reviews and Reports 22(5): 233-239, 2017.

20 Grabowski P, Schindler I, Anagnostopoulos I, Foss HD, Riecken EO, Mansmann U, Stein H, Berger G, Buhr HJ and Scherübl H: Neuroendocrine differentiation is a relevant prognostic factor in stage III-IV colorectal cancer. Eur J Gastroenterol Hepatol 13(4): 405-411, 2001.

21 Yang DK, Son CH, Lee SK, Choi PJ, Lee KE and Roh MS: Forkhead box M1 expression in pulmonary squamous cell carcinoma: correlation with clinicopathologic features and its prognostic significance. Hum Pathol 40(4): 464-470, 2009.

22 Fraedrich K, Schrader J, Ittrich H, Keller G, Gontarewicz A, Matzat V, Kromminga A, Pace A, Moll J, Bläker M, Lohse AW, Hörsch D, Brummendorf TH and Benten D: Targeting aurora kinases with danusertib (PHA-739358) inhibits growth of liver metastases from gastroenteropancreatic neuroendocrine tumors in an orthotopic xenograft model. Clin Cancer Res 18(17): 46214632, 2012.

23 Vandamme T, Peeters M, Dogan F, Pauwels P, Van Assche E, Beyens M, Mortier G, Vandeweyer G, de Herder WW, Van Camp G, Hofland L and Op de Beeck K: Whole exome characterization of pancreatic neuroendocrine tumor cell lines BON-1 and QGP-1. J Mol Endocrinol 54(2): 137-147, 2015.
24 Briest F, Grass I, Sedding D, Möbs M, Christen F, Benecke J, Fuchs K, Kaemmerer D, Mende S, Sänger J, Kunze A, Geisler C, Freitag H, Lewens F, Worpenberg L, Iwaszkiewicz S, Siegmund B, Walther W, Hummel M and Grabowski P: Mechanisms of targeting the MDM2-p53-FOXM1 axis in well-differentiated intestinal neuroendocrine tumors. Neuroendocrinology, 2017. doi: 10.1159/000481506. [Epub ahead of print]

25 Borisa AC and Bhatt HG: A comprehensive review on Aurora kinase: Small molecule inhibitors and clinical trial studies. Eur J Med Chem 140: 1-19, 2017.

26 Rodriguez JA, Lens SM, Span SW, Vader G, Medema RH, Kruyt FA and Giaccone G: Subcellular localization and nucleocytoplasmic transport of the chromosomal passenger proteins before nuclear envelope breakdown. Oncogene 25(35): 4867-4879, 2006.

27 Zaytsev AV, Segura-Pena D, Godzi M, Calderon A, Ballister ER, Stamatov R, Mayo AM, Peterson L, Black BE, Ataullakhanov FI, Lampson MA and Grishchuk EL: Bistability of a coupled Aurora B kinase-phosphatase system in cell division. Elife 5: e10644, 2016.

28 Rannou Y, Troadec MB, Petretti C, Hans F, Dutertre S, Dimitrov $\mathrm{S}$ and Prigent C: Localization of aurora $\mathrm{A}$ and aurora $\mathrm{B}$ kinases during interphase: role of the $\mathrm{N}$-terminal domain. Cell Cycle 7(19): 3012-3020, 2008.

29 Fazio N and Milione M: Heterogeneity of grade 3 gastroenteropancreatic neuroendocrine carcinomas: New insights and treatment implications. Cancer Treat Rev 50: 61-67, 2016.

Received May 9, 2018

Revised May 30, 2018

Accepted June 5, 2018 